\title{
Simulations of 3D observations of exoplanets using OWL
}

\author{
Alessandro Berton ${ }^{1}$, Markus Feldt ${ }^{1}$, \\ Raffaele G. Gratton ${ }^{2}$ and Thomas Henning ${ }^{1}$ \\ ${ }^{1}$ Max-Planck-Institut für Astronomie, Königstuhl 17, D-69117, Heidelberg, Germany \\ email: berton@mpia.de, feldt@mpia.de, henning@mpia.de \\ ${ }^{2}$ Osservatorio Astronomico di Padova INAF, Vic. Osservatorio 5, I-35122, Padova, Italy \\ email: gratton@pd.astro.it
}

\begin{abstract}
A software for simulations of observations with an Integral Field Spectrograph attached to an extreme adaptive optics system is presented here. This code, written in IDL, has been conceived within the CHEOPS project, a second generation "Planet Finder" instrument for ESO's VLT, and it has been successfully extended to the case of various ELTs in order to test the capabilities in detecting planets of an instrument based on Integral Field Spectroscopy (IFS). We present here, beside a general description of the procedure adopted to simulate IFS observations, a set of significant simulations of detections using OWL for different kinds of planets, in order to understand which objects are the best targets for an IFS-Planet Finder mounted on a $100 \mathrm{~m}$-class telescope.
\end{abstract}

Keywords. instrumentation: spectrographs - techniques: high angular resolution — planetary systems - telescopes

\section{Introduction}

Direct detections of Jupiter-like extrasolar planets, with present technology available and with present $10 \mathrm{~m}$-class telescopes, are a challenging goal to achieve. Difficulties come from the complex structure of the stellar Point Spread Function (PSF), which is covering the light of the planet. This stellar halo is usually brighter than the planet, at every wavelength, and atmospheric speckles are the most important source of noise that we have to consider in direct detections of planets (Racine et al. 1999). Beside an extreme AO system, which is mandatory in order to reduce dramatically the speckle intensity, additional techniques are required to reduce the speckle noise to a level comparable to the photon noise. Integral Field Spectroscopy (IFS) observations of the star-planet system have been suggested as a special Simultaneous Differential Imaging technique (SDI) within the CHEOPS project (CHaracterizing Exo-planets by Opto-infrared Polarimetry and Spectroscopy, Feldt et al. 2006), a "phase A" study for a Planet Finder instrument on ESO's VLT. The basic idea is to use the spectral features of the planet in the near-infrared, which are in general broad molecular absorption bands (e.g. methane $\left(\mathrm{CH}_{4}\right)$ bands for Jupiter-like planets, carbon dioxide $\left(\mathrm{CO}_{2}\right)$ for Venus-like ones, water for Earth-like planets), in order to enhance the planetary signal and then to subtract the light from the star and the resulting noise at the same time.

In order to test the capability of such a technique for reducing noise and detecting planets for various classes of telescopes, from 8 to 100 meters, we developed a dedicated software that simulates observations of extrasolar planets using an IFS. From the analysis of the results of the simulations, it has been possible to understand which classes of planets can be detected and characterized. In this paper the specific case of such an 

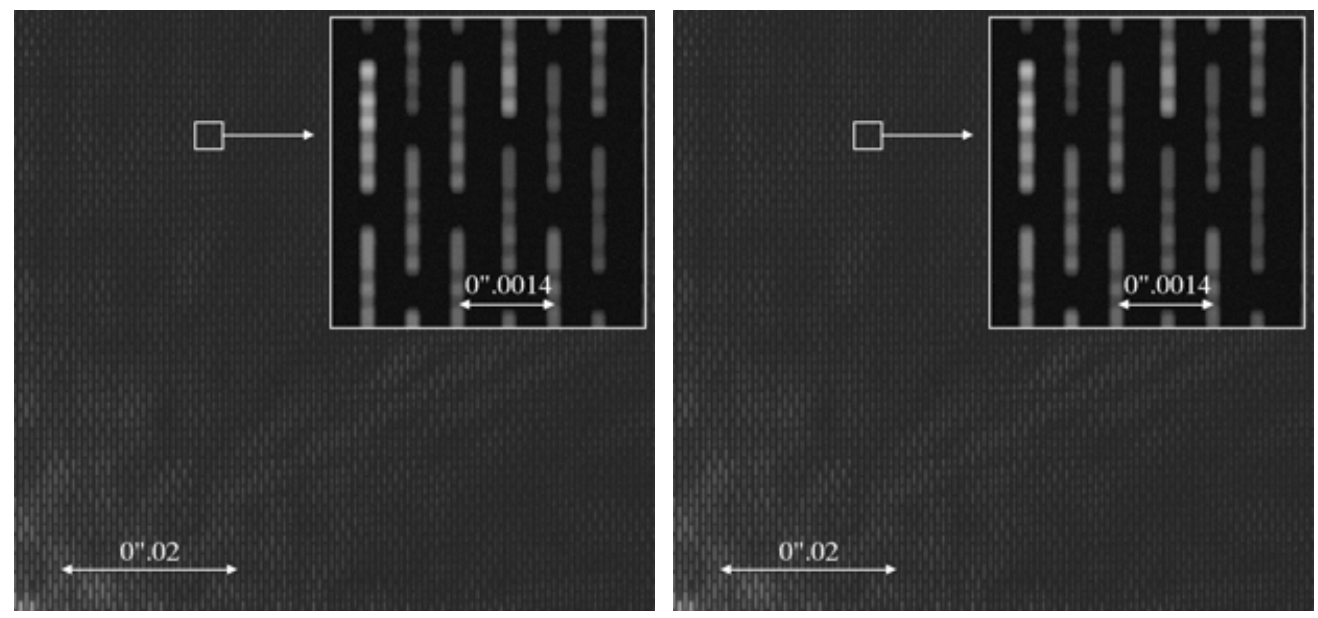

Figure 1. Left: Part of the final image of spectra as output of the simulation code. The insert shows the hexagonal configuration of spectra. The star is close to the left-bottom corner, outside the figure. Right: An example of detection, with a S/N 20, of a young Jupiter-like planet, visible in the $1^{\text {st }}$ quadrant of the image, at $1 \mathrm{AU}$ from its host star, located at $10 \mathrm{pc}$ from the Sun.

IFS-SDI instrument mounted on an OWL-type telescope (OverWhelmingly Large telescope, Gilmozzi 2004) is studied, and a compilation of expected integration times needed for the detection of four different planets is presented.

\section{Description of the simulation code}

The simulation code we are going to present here was written in IDL in the context of the CHEOPS project. The IFS consists of a microlens array composed of $254 \times 254$ hexagonal lenses covering a field of view of $0{ }^{\prime \prime} 35 \times 0$ "' 35 . The disperser is an Amici prism, which can give spectra with low and constant resolution in the spectral region between 0.9 and $2.0 \mu \mathrm{m}$. A detailed description of the CHEOPS IFS can be found in Claudi et al. (2004). The goal of our simulation code is to obtain an image of spectra as similar as possible to the one given by this IFS. In order to achieve this goal, we take great care in simulating the speckle patterns and to estimate the speckle noise correctly. Using these simulations (for an example of a simulated output image see the left part of Fig. 1), we studied the detectability of different classes of planets as a function of various parameters. Here we can distinguish the physical parameters of the planets (masses, ages, angular separations, distances), the instrumental parameters (telescope features, AO, etc.) and the atmospheric seeing conditions. A detailed description of the code will be available in a forthcoming paper (Berton et al. 2006b) and is summarized in Berton et al. (2006a). We used an entrance pupil for OWL, which takes into account the expected structure of spiders and the segmentation of primary and secondary mirrors (from Yaitskova et al. 2003); the performance of the AO system is simulated by creating a continuous series of phase screens each one separated from the others by an interval of time of $\sim 1 \mathrm{~ms}$, in order to simulate accurately the evolution of the speckles with time, corrected in real time by a system of 20000 actuators at a loop frequency of $500 \mathrm{~Hz}$. The resulting Strehl ratio is around 0.65 in $J$ band.

To reduce the "data" and to apply SDI we extract the information related to each specific wavelength from all the spectra. We create then a set of monochromatic images, each one corresponding to a given resolution element. These images, after an appropriate 
Table 1. Input parameters common to all the simulated cases

\begin{tabular}{lc}
\hline Telescope & OWL $(100 \mathrm{~m})$ \\
Strehl ratio & $\sim 0.65(J$-band) \\
Spectral resolution & 15 (Jupiter), 100 (Earth, Venus) \\
Separation & $1 \mathrm{AU}$ \\
Distance & $10 \mathrm{pc}$ \\
Stellar Type & G0V \\
\hline
\end{tabular}

scaling, can be combined in different ways, applying different mathematical operations in order to remove the stellar light and the speckles. Using only a subtraction between two wavelengths, on and off an absorption band, we can detect a planet with a certain signal-to-noise ratio (S/N, see, e.g., right part of Fig.1). It has been shown (Berton et al. 2006b) that the combination of up to 6 wavelengths, even better if they cover more than one planetary feature, can improve the final S/N significantly.

Such simulations would also be very useful for other ELT planet finder projects such as EPICS for OWL (Beuzit et al. 2006). We should note that the results presented here cannot be considered EPICS simulations, because the input parameters and requirements are slightly different. In our case, we followed a conservative approach starting from our present technical knowledge of AO, aberrations and sources of noise, coming from the experience at $8 \mathrm{~m}$-class telescopes: for this reason, for example, the used Strehl ratio of 0.65 is much smaller than the 0.91 , in $J$ band, required for EPICS. These differences are affecting our results significantly when compared with similar EPICS simulations.

\section{Simulated planetary detections}

We simulated four different cases: a very young (10Myr) and an old (1Gyr) Jupiter-like planet, an old Venus-like planet (5Gyr) and an Earth-like planet (5Gyr). For all these cases we used the same initial parameters (see Table 1) except for the spectral resolution $R$, which has to be tuned as a function of the spectral features we want to observe. In the case of Jupiter the most prominent features are the broad absorption bands of methane and for them a very low resolution $R=15$ is sufficient. For Venus we want to detect the narrower $\mathrm{CO}_{2}$ bands, and for them a higher resolution $(\sim 100)$ is suitable. In case of the Earth we used again $R=100$ and water bands, but additional studies have to be performed to show if low resolution is better to detect earth-like planets. Other examples of simulations with different telescope diameters are presented in Feldt et al. (2005).

\section{Results and discussion}

Results of our simulations, expressed in integration times needed to detect the planet with a $\mathrm{S} / \mathrm{N}$ equal to 5 are presented in Table 2 . In the first column the four cases are presented, while in the second one we listed the different molecules which characterize the planetary atmosphere and near-infrared spectra. Two methods have been used to enhance the planetary signal, as explained in Sec.2: a simple comparison of two wavelengths (results in the $3^{\text {rd }}$ column), on and off a specific absorption band, and an algorithm which combines up to 6 wavelengths on and off two different absorption bands (in the $4^{\text {th }}$ column). From these results, the gain in time that we can achieve using the second method appears to be of a factor of $\sim 3$.

The best case is a young Jupiter-like planet, visible clearly after few seconds of integration time. This is due to the strong thermal emission from the planet, which makes the 
Table 2. Integration time needed to detect the planet with $\mathrm{S} / \mathrm{N}=5$.

\begin{tabular}{l|c|cc}
\hline & Used features & Comparison of $2 \lambda$ & Comparison of $6 \lambda$ \\
\hline Young Jupiter (10Myr) & $\mathrm{CH}_{4}$ & $100 \mathrm{~s}$ & $30 \mathrm{~s}$ \\
Old Jupiter (1Gyr) & $\mathrm{CH}_{4}$ & $\sim 250 \mathrm{~h}$ & $\sim 60 \mathrm{~h}$ \\
Venus & $\mathrm{CO}_{2}$ & $\sim 300 \mathrm{~h}$ & $\sim 90 \mathrm{~h}$ \\
Earth & $\mathrm{H}_{2} \mathrm{O}$ & $\sim 1000 \mathrm{~h}$ & $\sim 300 \mathrm{~h}$ \\
\hline
\end{tabular}

object $\sim 150$ times brighter than the 1 Gyr case, characterized almost by reflected light. For the other cases, the observations require tens of hours, and in the case of the Earthlike planet the expected time is at best hundreds of hours. This makes the observation practically impossible. In this particular case it is important to note, anyway, that the water features we want to observe from the planet are exactly the same present in the Earth's atmospheric spectrum, and for this reason some corrections have been applied, adding noises in the data analysis phase.

From these numbers we can clearly see how difficult it would be to detect an old and cold extrasolar planets even using a 100m-class telescope like OWL, at least with the method suggested. This does not necessarily mean that the observation is impossible, but that the results strongly depend on the expected level of accuracy in handling instrumental sources of noise, and that observations would require a tremendous increase in AO performance. In fact, our simulations are based on some input parameters neither extremely optimistic nor pessimistic, because the AO correction, the static aberrations, the detector noise and in general all the instrumental features have been simulated based on the present experience with 8-10m-class telescopes. In the future, technology will probably enable to achieve more stable instruments with a better control of noise sources. For instance, our expected Strehl ratio is around 0.65 , but if it is possible in the future to reach a goal of 0.9 a coronagraph device can be considered in order to reduce significantly the photon noise and we expect in that case a reduction of the integration times needed by a factor of $\sim 10$.

In conclusion, to detect an Earth-like planet from the ground with IFS-SDI, within a reasonable integration time, we need an extreme accuracy in reducing noise and in controlling the whole instrument, much better than what can be done with the present technology.

\section{References}

Berton, A., Feldt, M., Gratton, R.G. et al. 2006a, in: Direct Imaging of Exoplanets: Science $\mathcal{G}$ Techniques; IAU Colloquium 200, 2005 October 3-7, Nice, France.

Berton, A., Gratton, R.G., Feldt, M. et al. 2006b, in preparation

Beuzit, J.L., Gratton, R.G., Kasper, M. et al. 2006, these proceedings

Claudi, R.U., Turatto, M., Gratton, R.G. et al. 2004, in: SPIE 5492, 1351

Feldt, M., Henning, T., Hippler, S. et al. 2006, in: Exploring the Cosmic Frontier: Astrophysical Instruments for the 21st century, ESO Astrophysics Symposia, in press

Feldt, M., Berton, A., Henning, T. \& Gratton, R. 2005, these proceedings

Gilmozzi, R. 2004, in SPIE 5489, 1

Racine, R., Walker, G.A.H., Nadeau, D. et al. 1999, PASP 111, 587

Yaitskova, N., Dohlen, K. \& Dierickx, P. 2003, in: SPIE 4840, 171 


\section{Discussion}

Crampton: Have you considered looking for emission lines instead of absorption features? All of us who have observed at northern latitudes know how strong such lines can be - although whether the NIR lines are strong is perhaps an issue.

BerTon: This idea is very interesting, but actually we worked, up to now, only with absorption bands. Emission lines would have for sure significant advantages and probably it will not be very difficult to study some examples in the next simulations.

QUIRRENBACH: (a) It seems that IFUs are better for spectral difference imaging than multi-channel cameras (such as SDI at NACO), because they minimize non-commonpath errors. (The spatial sampling is defined at the lenslet array, before the spectral channels are split). (b) It seems that the detection of cold planets from the ground will only be possible with AO system that achieve a performance sufficient for coronagraphs to work efficiently. Do you agree on these points?

Berton: Yes, I totally agree. The point (a) is one of the important advantages of integral field spectroscopy in detecting planets with simultaneous differential imaging. About the point (b), we couldn't simulate an appropriate coronagraph because our Strehl ratio is not very high, but its contribution in increasing the $\mathrm{S} / \mathrm{N}$ can be very significant.

Rouan: Do you assume an a priori knowledge of the spectrum in order to choose the two or six wavelengths? If yes, how do you get the information?

BERTON: One of the advantages of IFS is that it makes available information for many wavelengths. We want to develop an automatic procedure to compare all this information in all the possible ways, in order to enhance in the best way every spectral feature, wherever they are, and consequently to make a characterization of the planet.

DAVIDGE: Two questions: (1) Have you examined how detection time varies with Strehl ratio? This information would be useful to assess cost/performance trade-offs for planet search instruments on ELTs. (2) Have you included non-common path errors in your simulations?

A comment: Good talk!

Berton: (1) The variation of $\mathrm{S} / \mathrm{N}$ with the Strehl ratio has been studied theoretically, but specific simulation at different Strehl ratios have not been done. The reason is also the complexity of the method used to simulate AO in a very accurate way. (2) The simulation code has still some details that require to be studied better, and non-common path errors are within these details... (Comment by A. Quirrenbach: in any case an advantage of TIGER Type IFS is that it is optimized to reduce to a minimum level the non-common path errors!)

Thanks to Dr. Davidge for the comment!! 